\title{
DBT-informed treatment in a partial hospital and intensive outpatient program: the role of step-down care
}

\author{
Kirk D. Mochrie, ${ }^{1}$ John Lothes II, ${ }^{2}$ Eric Guender, ${ }^{3}$ Jane St. John ${ }^{3}$ \\ ${ }^{1}$ Triangle Area Psychology Clinic, Durham, NC; ${ }^{2}$ University of North Carolina Wilmington, Wilmington, NC; ${ }^{3}$ Delta Behavioral Health, \\ Wilmington, NC, USA
}

\begin{abstract}
Few studies to date have examined Partial Hospital (PH) and Intensive Outpatient (IOP) programs that utilize a Dialectical Behavior Therapy (DBT)-informed model. Preliminary findings suggest that DBT-informed PH programs are effective in reducing clinical symptoms; however, less is known about IOP programs as well as step-down care models. The present study utilized clinically relevant outcome indices and included a heterogeneous clinical sample. Specifically, the present study assessed pre-post data to examine changes in symptoms of depression, anxiety, hopelessness, and overall degree of suffering from intake to discharge in DBT-informed PH and IOP programs as well as a step-down condition (PH to IOP). Participants included 205 adults (ages $M=35.28, S D=12.49$ ). The sample was predominantly female $(\mathrm{N}=139,67.8 \%)$ and Caucasian $(\mathrm{N}=181,88.3 \%)$. The sample was divided into three distinct groups: PH program patients, PH to IOP program step-down patients, and IOP patients. Findings indicated significant symptom reduction from intake to discharge for all three conditions. There were no significant differences in mean change scores in symptom reduction between the three groups. Severity of depression symptoms at intake predicted program placement. However, type of program did not predict significant changes in symptoms from intake to discharge. This DBT-informed PH and IOP program was successful at reducing various psychiatric symptoms in the sample. Clinicians might consider the advantages of placing patients with higher symptoms of depression into $\mathrm{PH}$ programs with the intention of transitioning to step-down care through IOP programs that utilize DBT.
\end{abstract}

Key words: DBT-informed Treatment; Partial Hospital; Intensive Outpatient; Step-down Care; Symptom Reduction.

\section{Introduction}

Dialectical Behavior Therapy (DBT) is an intensive outpatient treatment for individuals with Borderline Personality Disorder (BPD) and contains multiple treatment

Correspondence: Kirk D. Mochrie, Triangle Area Psychology Clinic, Durham, NC 27713, USA.

E-mail: mochrie@tapclinicnc.com

Citation: Mochrie, K.D., Lothes II, J., Guender, E., St. John, J. (2020). DBT-informed treatment in a partial hospital and intensive outpatient program: the role of step-down care. Research in Psychotherapy: Psychopathology, Process and Outcome, 23(2), 109120. doi: 10.4081 /ripppo.2020.461

Conflict of interests: The Authors declare no potential conflict of interests.

Received for publication: 11 April 2020.

Accepted for publication: 21 May 2020.

This work is licensed under a Creative Commons Attribution NonCommercial 4.0 License (CC BY-NC 4.0).

${ }^{\circ}$ Copyright: the Author(s), 2020

Licensee PAGEPress, Italy

Research in Psychotherapy:

Psychopathology, Process and Outcome 2020; 23:109-120

doi:10.4081/ripppo.2020.461 components including individual therapy, group skills training, telephone coaching, and a consultation team (Linehan \& Wilks, 2015). Typically, a DBT skills group is two hours per week, with an individual therapy session lasting one hour per week. Many patients with BPD experience high levels of suicidal thinking. Therefore, one of the major aims of standard DBT is to reduce suicide attempts (Linehan, 1993; Tarrier, Taylor, \& Gooding, 2008). Moreover, DBT also aims to reduce Non-Suicidal Self-Injurious Behavior (NSIB), which is common among individuals with BPD (Linehan \& Wilks, 2015). Standard (comprehensive) DBT programs, are effective at targeting and reducing symptoms of emotion dysregulation and self-injurious behavior commonly associated with BPD (Tarrier et al., 2008).

In addition to BPD, research has also established the efficacy of using DBT to treat a variety of mental health disorders. However, most studies have examined individuals with BPD and other comorbid disorders. For example, research shows that standard DBT can be utilized to successfully treat BPD individuals with comorbid and significant eating disorders (Linehan \& Chen, 2005). There is also evidence for utilizing DBT combined with a Prolonged Exposure component for individuals with BPD comorbid PTSD (Harned, Wilks, Schmidt, \& Coyle, 2018). While research clearly demonstrates the efficacy of standard DBT programs, less is known about DBT-informed treatment.

DBT-informed programs utilize specific components of DBT; however, may not all of the standard treatment 
modes (e.g., individual therapy, skills group, coaching phone, treatment team). Interestingly, Panos and colleagues conducted a meta-analysis which indicated that there is good preliminary evidence accumulating to suggest the effectiveness of DBT-informed treatment for various mental health disorders (Panos, Jackson, Hasan, \& Panos, 2014). For example, DBT-informed treatment is effective at treating both anxiety and depressive symptoms (Lothes, Mochrie, \& St. John, 2014) common among internalizing disorders. There is also literature supporting the use of DBT-informed programs to treat Substance Use Disorders, specifically symptoms of emotion dysregulation related to alcohol dependence (Maffei, Cavicchioli, Movalli, Cavallaro, \& Fossati, 2018). Studies have also demonstrated the effectiveness of DBT-informed treatment with individuals with bipolar disorder, primarily by increasing coping skills and decreasing associated emotion dysregulation (Eisner et al., 2017). In addition, there is some preliminary research suggesting that DBT skill modules can be used as a stand-alone treatment for depressive disorders (Valentine, Bankoff, Poulin, Reidler, \& Pantalone, 2015). Similarly, some evidence indicates that only using a subset of all the components of comprehensive DBT (i.e., only individual therapy and skills group) is effective in treating eating disorders (Telch, Agras, \& Linehan, 2001). There is also some initial evidence in support of using specific DBT skill modules in lieu of the full treatment package to help reduce emotion dysregulation (Neasciu, Eberle, Kramer, Wiesmann, \& Linehan, 2014). Taken together, these findings suggest that DBT-informed treatment can be effective at treating various mental health disorders/symptoms.

The label of DBT-informed treatment is also used when DBT has been adapted within a different treatment setting than what was used in prior RCT studies of its efficacy. Interestingly, there is some preliminarily research on such adaptations. For example, inpatient DBT programs are often used when an individual is hospitalized for active suicidal thinking and behaviors as well as attempts. Given that DBT is considered a suicide prevention modality to a life worth living (Chapman, 2019a; Linehan, 1993; Linehan \& Wilks, 2015), it makes sense to adapt it for inpatient settings with highly suicidal individuals, which seems to be becoming more popular. For example, Kröger, Harbeck, Armbrust, and Kliem (2013) found that adapting DBT for an inpatient setting among individuals with BPD was effective at reducing various symptoms of the disorder, including suicidal thoughts and behavior from intake to discharge. However, inpatient stays are often brief, and patients may struggle to acquire all the skills from the DBT modules during such a short duration. Instead, many patients are referred to Partial Hospital $(\mathrm{PH})$ or Intensive Outpatient (IOP) programs upon discharging from an inpatient stay (Neuhaus, 2006). Many $\mathrm{PH}$ and IOP programs utilize $\mathrm{CBT}$ as the main treatment modality. While this is an effective treatment modality for a variety of mental health disorders, it may not be the most useful for individuals with high suicidal ideation/behaviors who would likely benefit more from an adaptation of DBT in such programs (Panos et al., 2014).

IOP programs typically meet 3 days per week for group therapy (two to three hours each day) as well as include individual therapy, typically meeting once a week for approximately one hour per session. There is currently a dearth of research on DBT-informed IOP programs. Interestingly, one study used a blend of DBT and CBT strategies to treat individuals with eating disorders, with results demonstrating significant reductions in eating disordered symptoms, suicidal and self-injurious behaviors, treatment interfering behaviors, psychiatric and medical hospitalizations, and clinician burnout (Federici \& Wisniewski, 2013). However, there is a complete lack of research on utilizing a DBT-only approach in an IOP program, with one exception. Ritschel and colleagues examined changes in depression, anxiety, hope, and mindfulness among 56 individuals in a DBT-informed IOP program located within a community mental health center (Ritschel, Cheavens, \& Nelson, 2012). Of note, $80 \%$ of the sample met criteria for a mood disorder, approximately $50 \%$ met criteria for an anxiety disorder, and $20 \%$ met criteria for comorbid substance abuse/dependence and the average length of stay in the program was six weeks. Findings revealed significant decreases in depression and anxiety as well as increases in hope scores throughout the duration of the treatment. These results provide preliminary evidence for the effectiveness of adapting DBT in an IOP program; however, more research is needed to replicate these findings.

In terms of PH programs, there is some preliminary evidence that DBT can be an effective treatment when utilized in this setting, specifically for individuals with BPD (Lenz \& Del Conte, 2018). Other research has demonstrated the effectiveness of DBT-informed treatment within a PH setting to reduce symptoms of depression, anxiety, hopelessness, and overall suffering among a sample of individuals with MDD, GAD, PTSD, and bipolar disorders (Lothes et al., 2014). However, PH programs are often short in duration and many patients need further treatment after discharge. Importantly, a more recent study on a DBT-informed PH program by Lothes and colleagues examined various symptoms in relation to treatment duration among 113 adults with similar mental health diagnoses reported in their original study who spent an average of 23 days in the program. Findings revealed that patients with a higher level of symptom acuity at intake in combination with those who were able to attend the program for a longer duration achieved greater reductions in various clinical symptoms from intake to discharge (Lothes, Mochrie, Quickel, \& St. John, 2016).

Importantly, these findings do suggest that while DBT can be effectively adapted for different treatment settings such as IOP and PH programs; however, there remains a lack of research in this area. 
In addition, many treatment facilities utilize step-down care which reduces the number of face-to-face contact hours for overall treatment. Step-down care involves referring a patient to a lower level of care (i.e., step-down) rather than discharging them from treatment services. Specifically, patients who demonstrate improvement and reduced symptoms in a higher level of care who still necessitate some treatment, might be referred to step-down care programs (e.g., a PH to an IOP program). There are very few studies that directly examine the role of stepdown care in such settings. However, Antonsen et al. (2014), conducted an RCT with 113 psychiatric patients with personality disorders to compare outpatient individual psychotherapy with long-term step-down treatment within a program that included a short-term day hospital treatment followed by combined group and individual psychotherapy. Findings showed that between the 3 and 6-month follow up points of data collection, participants in the individual therapy only group demonstrated significant declines in psychosocial functioning compared to individuals in the step-down care group suggesting that step-down care may be important in maintain treatment progress.

Of note, the role of step-down care for treatment seeking individuals entering DBT-informed $\mathrm{PH}$ programs has not been examined to date. Most studies on DBT tend to assess individuals with BPD (Stoffers et al., 2012), rather than examining heterogenous diagnostic samples. The present study examined data on a DBT-informed $\mathrm{PH}$ and IOP program among patients with a variety of mental health diagnoses and included some specific analyses to assess step-down care (i.e., PH to IOP) among these patients. A general description of the DBT-informed program and DBT adherence as well as the specifics of the $\mathrm{PH}$ and IOP programs are outlined below.

\section{General DBT-informed Program Description and DBT Adherence}

Research has demonstrated that the most effective ways to implement DBT include therapists who consistently assess for DBT skills acquisition, strengthening, and generalization at the different stages of treatment (Swales \& Dunkley, 2019). Swales argues that this implementation is critical when working with highly suicidal individuals to maintain skill use targeting a reduction of these symptoms on a regular basis. The present study ensured that all clinicians who treated DBT patients engaged in this practice. Moreover, consistent assessment of barriers preventing effective skill use combined with problem solving was used by all clinicians within the DBT-informed programs, consistent with the current DBT literature (Swales \& Dunkley, 2019). Standard DBT was adapted to the PH and IOP programs in this study. Importantly, both the PH and IOP programs met and adhered to all 5 principles of DBT (Linehan, 1993). The adaptations of the protocol for applying DBT in each program allowed both the PH and IOP programs to remain adherent in the application of DBT. Moreover, DBT adherence has been shown in other therapeutic modalities (Swenson, 2016), with one of the key features being that the modes and functions of DBT are met as was ensured in the programs utilized in this study. Both programs included: DBT skills training, individual psychotherapy, in-the-moment skills coaching, case management, and weekly DBT treatment team meetings. Moreover, through these modalities the following DBT functions were met by the programs: improving patient motivation, generalization of skills, structuring the environment, and enhancing therapist capabilities and supporting motivation.

Patients enrolled in the $\mathrm{PH}$ and IOP programs also received weekly DBT individual therapy which complied with standard DBT requirements within each session by starting with diary card review, attention to the treatment hierarchy, chain analysis on the highest target behaviors with solution analysis, and skills generalization by moving down the hierarchy to the patients use of skills in current life situations. In addition, secondary treatment targets were discussed, and therapists used dialectical strategies throughout their individual treatment sessions, consistent with DBT adherent recommendations (Koerner, 2012). Patients were also given 24/7 coaching phone access. In addition, there was a weekly 1.5 -hour DBT treatment team meeting that utilized the DBT agenda and roles required in standard DBT.

The programs described below also aimed to help individuals reduce symptoms of depression, anxiety, and hopelessness by increasing emotion regulation skills and utilizing a variety of DBT change strategies in both skills group and individual therapy. During the orientation process, patients were first introduced to the model for describing emotions as well as the standard DBT commitments. The Biosocial model of DBT (Chapman, 2019a; Linehan, 1993) was also discussed at intake and during weekly skills groups to help patients gain insight as to how their biology and prior experiences of invalidation within their environments have influenced their ability to effectively regulate emotions. A sample week in both the $\mathrm{PH}$ and IOP program is provided in Table 1. Of note, both programs were flexible in terms of treatment duration based on individual patient needs and treatment progress.

\section{PH Program Description}

The PH program of study is considered an intensive outpatient day treatment option for adults with acute mental illness that has adapted DBT constructed from Linehan's DBT training manual, Second Edition (Linehan \& Wilks, 2015). This specific community mental health center conducts ongoing program evaluation aimed at increasing the fidelity of DBT adherence and examining treatment outcomes. The $\mathrm{PH}$ program patients met 5 days a week for 4 hours each day. Treatment included skills training in mindfulness, emotion regulation, distress tol- 
erance, and interpersonal effectiveness as well as process groups, individual therapy, 24-hour coaching phone consultation, DBT team meetings, medication management, and psychiatric care when necessary.

Patients in the $\mathrm{PH}$ program attended four separate groups each program day. The first group began with a "check-in" process that included homework review and problem solving any barriers to implementing skills outside of group. The second and third groups included 50min skills teaching sessions with time for questions and the assignment of skills homework. Typically, one to two DBT skills were taught in each group; the skills modules rotated so that each group day incorporated skills from a different module. This format covered all DBT skills in a six-week time period. The final (fourth) group was used to address potential barriers to utilizing skills before returning to the next program day. A brief mindfulness practice was also implemented each day in the program and typically occurred during the check-in process of the first hour of group, unless mindfulness skills were being taught during the skills group that day, in which case the practice would take place during those specific group times. In addition, patients met several times per week with a nurse and once per week with a medical doctor for medication management. Patients were also encouraged to call a 24- hour coaching phone to decrease suicidal behaviors and promote DBT skill use outside of group (Ben-Porath \& Koons, 2005; Chapman, 2019b).

Moreover, patients met with a weekly individual therapist who was trained in DBT to work on specific behavioral treatment targets. Any patient with suicidal thinking or NSIB had a treatment target of reducing these through DBT skill use. Of note, there were at least three 10-day DBT intensively trained therapists and at least five, 5-day DBT foundationally trained therapists on staff during the study. Therapists and interns who had not attended a 10 or 5-day intensive training yet belonged to the DBT consultation team as well as received weekly individual supervision from one of the intensively trained therapists.

\section{IOP Program Description}

The IOP program mimicked the $\mathrm{PH}$ program in many ways; however, met less frequently. Specifically, patients in the IOP program met for three days a week, three hours per day for group. The groups began with the same checkin process used for the PH program. The second hour and part of the third hour of each group was spent reviewing homework and teaching skills. The last part of the final hour of each group was used to teach a new skill and al-

Table 1. Sample 1-week Schedule for PH and IOP programs.

\begin{tabular}{|c|c|c|}
\hline & PH program (4hrs per day) & IOP program (3hrs per day) \\
\hline \multirow[t]{4}{*}{ Mon } & Check In-process/ Homework Review & Check In-process/Homework Review \\
\hline & ER: Goals/What Emotions Do for You & ER: Goals/What Emotions Do for You \\
\hline & ER: Model for Describing Emotions & ER: Model for Describing Emotions \\
\hline & Goals of Skills Training & \\
\hline \multirow[t]{4}{*}{ Tue } & Check In-process/ Homework Review & \\
\hline & Diary Cards & \\
\hline & Dialectics & \\
\hline & MI: A Day Away from Stress & \\
\hline \multirow[t]{4}{*}{ Wed } & Check In-process/ Homework Review & Check In-process/ Homework Review \\
\hline & IE: Goals/Factors in the Way of IE-Do Myths worksheet in group & IE: Goals/Factors in the Way of IE \\
\hline & MI: Goals of Mindfulness & MI: Goals of Mindfulness \\
\hline & Guidelines for Skills Training/Skills Training Assumptions & \\
\hline \multirow[t]{4}{*}{ Thu } & Check In-process/Homework Review & \\
\hline & Thought Records & \\
\hline & Chain Analysis-Missing Link Analysis & \\
\hline & MI: 10-minute Sitting Meditation & \\
\hline \multirow[t]{4}{*}{ Fri } & Check In-process/Homework Review & Check In-process/Homework Review \\
\hline & DT: Goals/When to Use Crisis Survival Skills & DT: Goals/When to Use Crisis Survival Skills \\
\hline & DT: STOP skills/Pros and Cons & DT: STOP skills/Pros and Cons \\
\hline & Biosocial Theory & \\
\hline
\end{tabular}

Note. Blank sections within the right (IOP) column of the table were days/times that the group did not meet.

$\mathrm{ER}=$ Emotion Regulation; DT $=$ Distress Tolerance; $\mathrm{IE}=$ Interpersonal Effectiveness; $\mathrm{MI}=$ Mindfulness . 
lowed time for group process/questions. When mindfulness was not scheduled as a specifically taught skill that day, mindfulness practices were completed after the first group check-in and before the beginning of the first skills group for the day. If mindfulness was taught as a specific skill that week, then a mindfulness practice was conducted during that group. This sometimes resulted in mindfulness being done at the end of the program day to align with the skills teaching during that group hour. Patients also received access to phone coaching, and appointments with a prescriber for medication management if needed. Individual DBT weekly therapy was also required and utilized the DBT treatment hierarchy in addressing treatment targets and patient goals. Overall, the only difference between the PH and IOP program was the amount of time spent in group each week which resulted in covering all DBT skills in a 12-week span.

\section{Step-down Care (PHP-IOP)}

Patients in this program were first enrolled in the $\mathrm{PH}$ program before entering the IOP program. Everything else remained the same. The clinic's DBT treatment team met and discussed which patients would benefit from engaging in step-down care based on their treatment progress (i.e., number of days in $\mathrm{PH}$ program, suicidal actions/thoughts, NSIB actions/urges, severity of symptomatology, participation, attendance, and demonstration of skill use).

\section{Purpose of the Present Study}

There are a limited number of studies to date that have examined DBT IOP programs, with some preliminary results suggesting efficacy for these programs in reducing various symptoms (Ritschel et al., 2012). There is no data on differences between DBT-informed PH and IOP programs in terms of treatment outcomes. Thus, the present study examined a large sample of patients in a community mental health clinic to assess changes in symptoms of depression, anxiety, hopelessness, and overall degree of suffering from intake to discharge in three different conditions; those that attended a DBT-informed PH program only, those that stepped-down from PH to IOP, and those that attended the IOP program only. The present study uniquely adds to the existing literature by being the first to measure these outcomes within a DBT-informed $\mathrm{PH}$, step-down, and IOP program in the southeast region of the United States. In addition, the present study sought to examine the relationship of the number of overall hours spent in treatment from intake to discharge to changes in symptoms. Finally, differences among programs in terms of outcomes and time in treatment were examined.

It was hypothesized that in the PH, IOP, and stepdown program conditions there would be significant reductions in all symptom scores (i.e., depression, anxiety, hopelessness, and overall suffering) from intake to dis- charge. Next, it was hypothesized that there would be no significant differences in symptom changes based on the type of program (i.e., PH, IOP, step-down) that participants were enrolled in. In addition, it was hypothesized that program type would relate to overall hours in treatment; specifically, that those in the step-down (PH to IOP) program would receive significantly more overall hours of treatment than those that only participated in the $\mathrm{PH}$ or IOP programs. It was also hypothesized that regardless of the program type, participants who engaged in a greater number of overall hours of treatment would also show greater reductions in symptoms (i.e., depression, anxiety, hopelessness, overall suffering) from intake to discharge. Finally, it was hypothesized that individuals in the $\mathrm{PH}$ and step-down programs would have significantly higher symptom scores (i.e., depression, anxiety, hopelessness, overall suffering) at their initial intake assessment than those in the IOP program.

\section{Method}

\section{Participants}

Participants included 205 adults split into three groups; those that only attended the $\mathrm{PH}$ program $(\mathrm{N}=33)$ ages 18-57 $(M=35, S D=11)$, predominantly female $(N$ $=25)$ and Caucasian $(N=27)$; those that started in PHP and stepped-down to the IOP program $(\mathrm{N}=106)$, ages 19$65(M=36, S D=12)$, predominantly female $(N=72)$ and Caucasian $(N=88)$; those that only attended the IOP program $(\mathrm{N}=66)$, ages 18-66 $(M=34, S D=13)$, predominantly female $(N=42)$ and Caucasian $(N=64) . S D$. Since this was an assessment of data collected from patient records and was not an RCT, structured clinical interviews were not conducted at intake. Therefore, many of the patients had co-occurring diagnoses (e.g., BPD and PTSD) that were not recorded due to only primary diagnoses being recorded for data analysis. Primary diagnoses of the sample included depressive disorders $(\mathrm{N}=97)$, bipolar I and II disorders $(\mathrm{N}=43)$, Substance Use Disorders $(\mathrm{N}=$ 29), and PTSD $(\mathrm{N}=9)$. In addition, there were a small number of participants $(\mathrm{N}=14)$ who had primary diagnoses of ADHD, adjustment disorder, anxiety disorders, psychotic disorders, and unspecified illness that were included in the sample. Further, 11 participants were given a code for diagnosis deferred at intake and these were not updated before they discharged. Finally, two individuals were given the diagnosis of an anxiety-related medical disorder.

\section{Materials}

\section{Depression}

The Clinically Useful Depression Outcome Scale (CUDOS; Zimmerman \& McGlinchey, 2008) was used 
to assess self-reported ratings of depression at intake and discharge due to its clinical utility in treatment planning. This scale is brief, easy to administer and score, and fully covers the Diagnostic and Statistical Manual of Mental Disorders, fourth edition (DSM-IV-TR) symptoms for MDD. Good reliability (Cronbach's alpha $=.90$ ) and validity have been established using this measure with psychiatric patients (Zimmerman, Chelminski, McGlinchey, $\&$ Posternak, 2008). Due to the high number of individuals with depression symptoms in the present study, this measure was chosen to best capture those symptoms in relation to clinical treatment planning. Responses to specific items were useful in helping individual therapists develop treatment targets for their patients.

\section{Anxiety}

The Clinically Useful Anxiety Outcome Scale (CUXOS; Zimmerman, Chelminski, Young \& Dalrymple, 2010) was used to assess self-reported ratings of anxiety at intake and discharge due to its clinical utility in treatment planning. Similar to the CUDOS, the CUXOS is a brief tool that easy to administer and score and has been shown to have good reliability (Cronbach's alpha $=.90$ ) and validity when assessing for anxiety symptoms and clinically significant symptom change among psychiatric patients (Zimmerman et al., 2010; Jeon et al., 2017). Due to the high number of individuals with anxiety symptoms in the present study, this measure was chosen to best capture those symptoms in relation to clinical treatment planning. Responses to specific items were useful in helping individual therapists develop treatment targets for their patients.

\section{Hopelessness}

The Beck Hopelessness Scale (BHS; Beck \& Steer, 1988) was used to assess self-reported levels of hopelessness at intake and discharge. The BHS is a 21-item measure that assesses hopelessness, which is related to both depression and anxiety. It is considered a reliable and valid measure with good internal consistency (Cronbach's alpha $=.97$ ) among depressed patients (Bouvard, Charles, Guerin, Aimard, \& Cottraux, 1992). This measure was chosen due to its clinical utility for individual DBT therapists to assess and target high levels of hopelessness. Within the present sample, the BHS was used to ensure that the individual therapists were utilizing DBT orientation and commitment strategies to specifically target decreasing hopelessness and increasing willingness for treatment commitment.

\section{Suffering}

Self-reported overall degree of suffering was measured using a Likert scale ranging from 0 (No suffering at all) to 10 (Worst suffering ever) and administered at intake and discharge. While this measure has not been validated against other measures of suffering, it was used to help determine overall distress/suffering at intake as one of several previously mentioned factors that was considered in treatment allocation decisions.

\section{Hours in Treatment}

Overall hours in treatment encompassed the total number of hours spent in both group and individual therapy from intake to discharge based on attendance for each patient.

\section{Procedure}

The study was approved by an Institutional Review Board at a local university. All participants were evaluated for medical necessity during intake and again at discharge. Individuals who presented with significant suicidal thinking and NSIB who had a recent (within the last month) inpatient hospitalization (for this or a suicide attempt) were admitted to the PH program. In addition, severity of depression and anxiety scores and an individual's ability to complete activities of daily living were considered when making all program placements. In contrast, individuals admitted to the IOP program, typically reported less severe suicidal thinking as well as NSIB and were not hospitalized within the last month for safety concerns or a suicide attempt. These individuals also presented with more moderate symptoms of depression and anxiety compared to severe symptoms and were generally able to complete most activities of daily living. Participants did not receive compensation for participation in the study. All patients signed a written informed consent for treatment. Assessment measures described in the measures section were administered at intake and at the time of each patient's discharge from programming.

\section{Data Analysis}

Analyses were conducted using SPSS - Version 25, Greenville, NC and raw scores were chosen for analyses in order to optimize clinical utility. A series of repeated measures ANOVAs were conducted to examine changes in symptom scores from intake to discharge for the PH, IOP, and step-down care treatment conditions. Difference scores were calculated for all of the symptom measures from intake to discharge. These scores were compared using between subjects' one-way ANOVAs to examine any differences in change from intake to discharge based on program type (i.e., PH, IOP, and Step-down). A series of linear regression analyses were conducted to examine the relationship of overall hours in treatment to difference scores among all the symptom variables. A one-way ANOVA was also conducted to examine differences in overall treatment hours based on program type. Finally, a MANCOVA was used to examine any differences in symptom scores at intake based on program type, controlling for overall hours in treatment to examine what proportion of the variance in symptom scores was accounted for by program type. A G-power analysis was conducted 
for the MANCOVA analysis with an alpha of .05, effect size of .30 , and with $80 \%$ power due to this analysis needing the highest total sample size. Results of this analysis showed that a total sample size of 190 participants was needed, suggesting the present study sample of 205 individuals was adequate to proceed with the analysis.

\section{Results}

\section{Differences Between Programs in Symptom Reduction}

Results showed a significant decrease in depression scores for participants in the $\mathrm{PH}$ program from intake $(M$ $=35.74, S D=14.74)$ to discharge $(M=21.82, S D=$ $15.80), F(1,32)=23.51, p<.0001$. There was also a significant reduction from intake $(M=39.03, S D=15.49)$ to discharge $(M=18.99, S D=15.80), F(1,105)=166.62, p$ $<.0001$, among patients who began in the $\mathrm{PH}$ program and then stepped-down into the IOP program. Finally, patients in the IOP only program showed significant reductions in depression symptoms from intake $(M=29.49, S D$ $=15.55)$ to discharge $(M=12.77, S D=13.44), F(1,65)$ $=58.06, p<.0001$. Figure 1 visually displays these results. There were no significant differences between depression change scores in the $\mathrm{PH}$ program $(M=17.13, S D$ $=13.61)$ compared to step-down care $(M=22.17, S D=$ $14.04)$ and the IOP program $(M=19.91, S D=12.16), F$ $(2,202)=2.09, p=.13$. This was also true when comparing scores between the IOP program and step-down care.

Results showed a significant decrease in anxiety scores for participants in the $\mathrm{PH}$ program from intake $(M$ $=39.67, S D=18.72)$ to discharge $(M=26.85, S D=$ $18.45), F(1,32)=12.70 p<.001$. There was also a significant reduction from intake $(M=41.24, S D=21.21)$ to discharge $(M=23.73, S D=17.82), F(1,105)=91.21, p$ $<.0001$, among patients who began in the PH program and then stepped-down into the IOP program. Finally, patients in the IOP only program showed significant reductions in anxiety symptoms from intake $(M=33.65, S D=$ $19.47)$ to discharge $(M=17.80, S D=14.61), F(1,65)=$ $36.27, p<.0001$. Figure 2 visually displays these results. There were no significant differences between depression change scores in the PH program $(M=17.90, S D=14.73)$ compared to step-down care $(M=20.75, S D=15.87)$ and the IOP program $(M=20.41, S D=17.15), F(2,202)=$ $0.46, p=.63$. This was also true when comparing scores between the IOP program and step-down care.

Results showed a significant decrease in hopelessness scores for participants in the $\mathrm{PH}$ program from intake $(M$ $=11.03, S D=5.79)$ to discharge $(M=7.42, S D=6.13)$, $F(1,32)=22.91, p<.0001$. There was also a significant reduction from intake $(M=11.78, S D=6.19)$ to discharge $(M=6.97, S D=5.79), F(1,105)=61.66, p<.0001$, among patients who began in the $\mathrm{PH}$ program and then stepped-down into the IOP program. Finally, patients in the IOP only program showed significant reductions in hopelessness symptoms from intake $(M=8.06, S D=$ $5.70)$ to discharge $(M=3.68, S D=3.45), F(1,65)=$ $48.34, p<.0001$. Figure 3 visually displays these results. There were no significant differences between depression change scores in the PH program $(M=5.08, S D=4.18)$ compared to step-down care $(M=5.65, S D=5.18)$ and the IOP program $(M=5.33, S D=4.61), F(2,202)=0.22$, $p=.80$. This was also true when comparing scores between the IOP program and step-down care.

Results showed a significant decrease in overall suffering scores for participants in the $\mathrm{PH}$ program from intake $(M=7.03, S D=2.26)$ to discharge $(M=4.00, S D=$

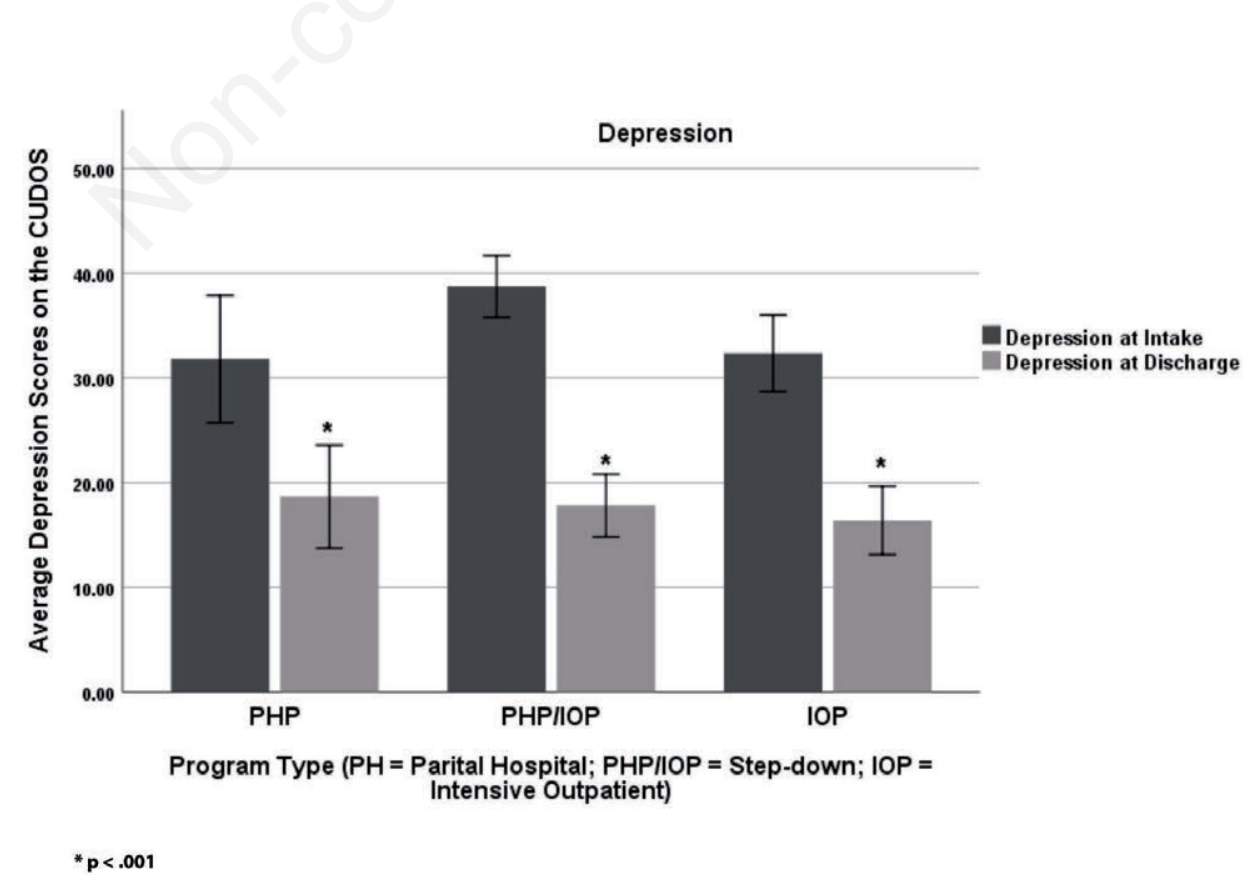

Figure 1. Average Depression Scores from Intake to Discharge by Program Type. 
$2.95), F(1,32)=38.95, p<.0001$. There was also a significant reduction from intake $(M=7.20, S D=2.33)$ to discharge $(M=3.24, S D=2.65), F(1,105)=216.70, p<$ .0001 , among patients who began in the PH program and then stepped-down into the IOP program. Finally, patients in the IOP only program showed significant reductions in hopelessness symptoms from intake $(M=5.82, S D=$ $2.58)$ to discharge $(M=1.89, S D=2.06), F(1,65)=$ $138.61, p<.0001$. Figure 4 visually displays these results. There were no significant differences between depression change scores in the $\mathrm{PH}$ program $(M=3.38, S D=2.66)$ compared to step-down care $(M=4.05, S D=2.64)$ and the IOP program $(M=4.02, S D=2.45), F(2,202)=1.01$, $p=.37$. This was also true when comparing scores between the IOP program and step-down care.

\section{The Role of Overall Hours of Treatment in Predicting Symptom Reduction}

Linear regression analysis revealed that overall hours in treatment did not predict changes in depression from intake to discharge, $F(1,196)=1.69, p=.195$. This was

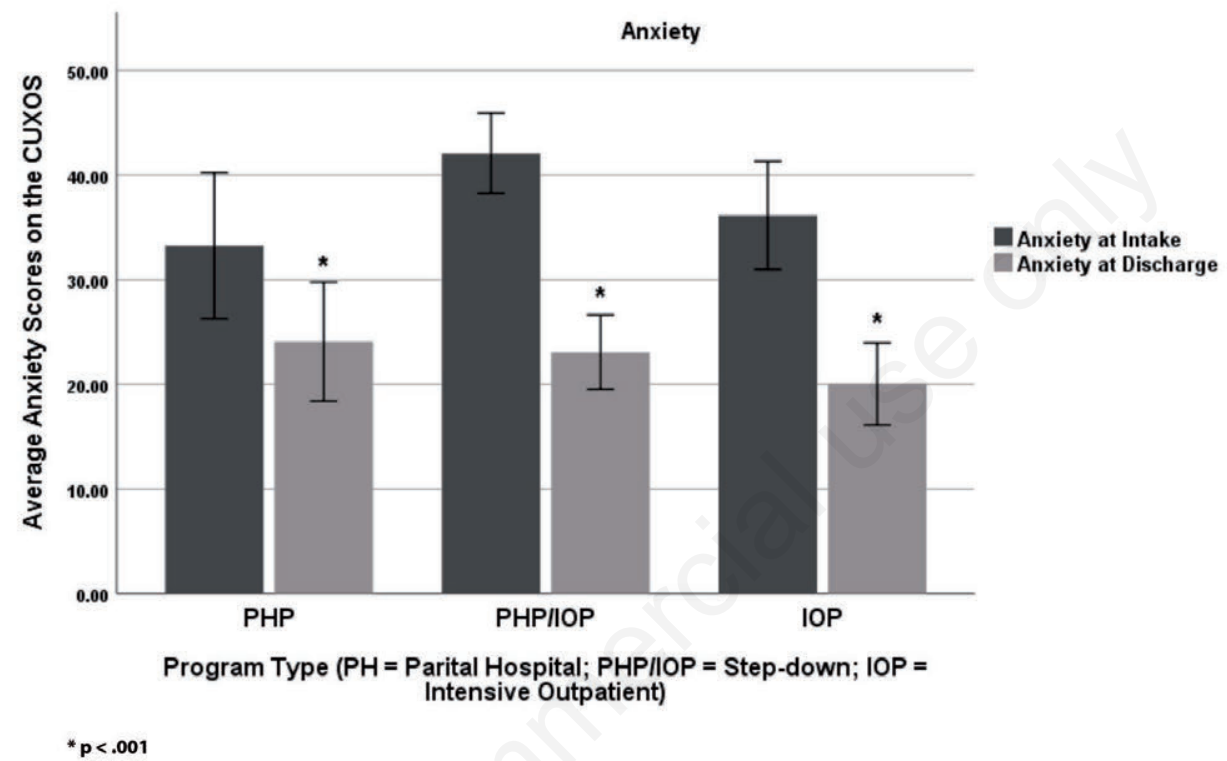

Figure 2. Average Anxiety Scores from Intake to Discharge by Program Type.

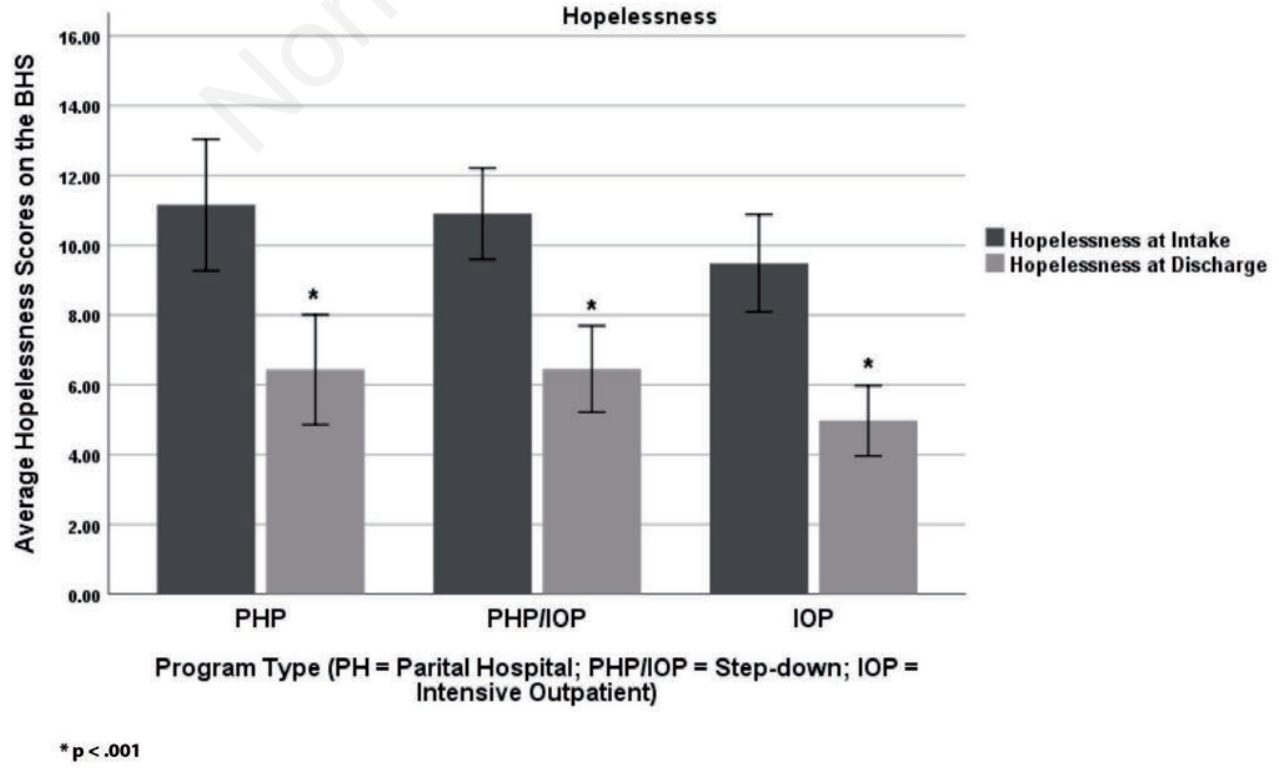

Figure 3. Average Hopelessness Scores from Intake to Discharge by Program Type. 
also true for predicting changes in anxiety, $F(1,196)=$ $0.001, p=.982$, hopelessness, $F(1,196)=0.15, p=.70$. and overall suffering, $F(1,196)=0.96, p=.33$, within the sample.

\section{Differences Between Programs in Hours of Treatment}

There were significant differences in overall hours in treatment based on program. Specifically, individuals in the step-down care condition engaged in significantly more overall hours of treatment $(M=223.14, S D=$
180.17) compared to those in the $\mathrm{PH}$ program $(M=$ $104.27, S D=119.19)$ and those in the IOP program $(M=$ $42.53, S D=45.38), F(2,195)=32.49, p<.0001$. Figure 5 visually displays these results. Lastly, a MANCOVA, controlling for overall hours of treatment and using a Bonferroni posthoc analysis, revealed that individuals with higher depression scores at intake were significantly more likely to be placed in the PH to IOP step-down care condition compared to scores among those in the IOP program, $F(2,201)=5.61, p<.05,(M$ diff $=6.38, S E=2.49$, $p=.03)$. Further, those with higher depression scores at

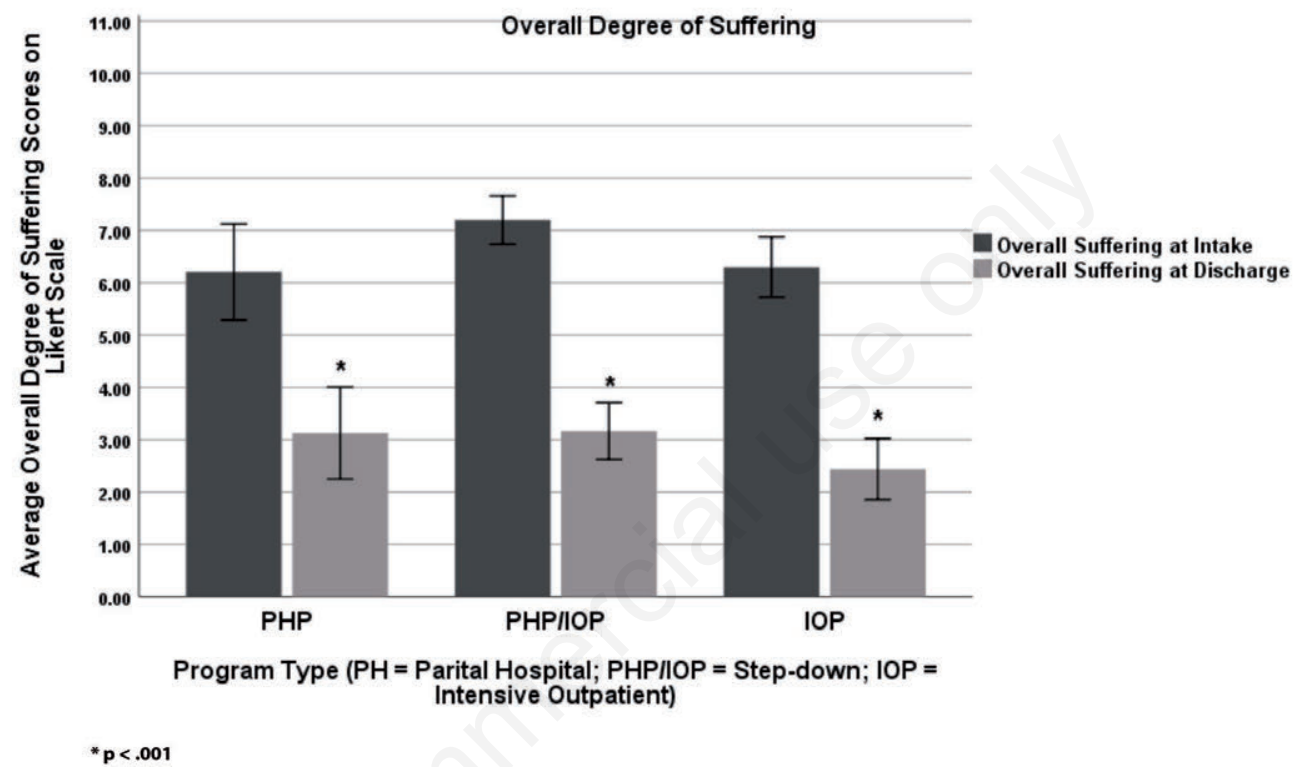

Figure 4. Average Overall Suffering Scores from Intake to Discharge by Program Type.

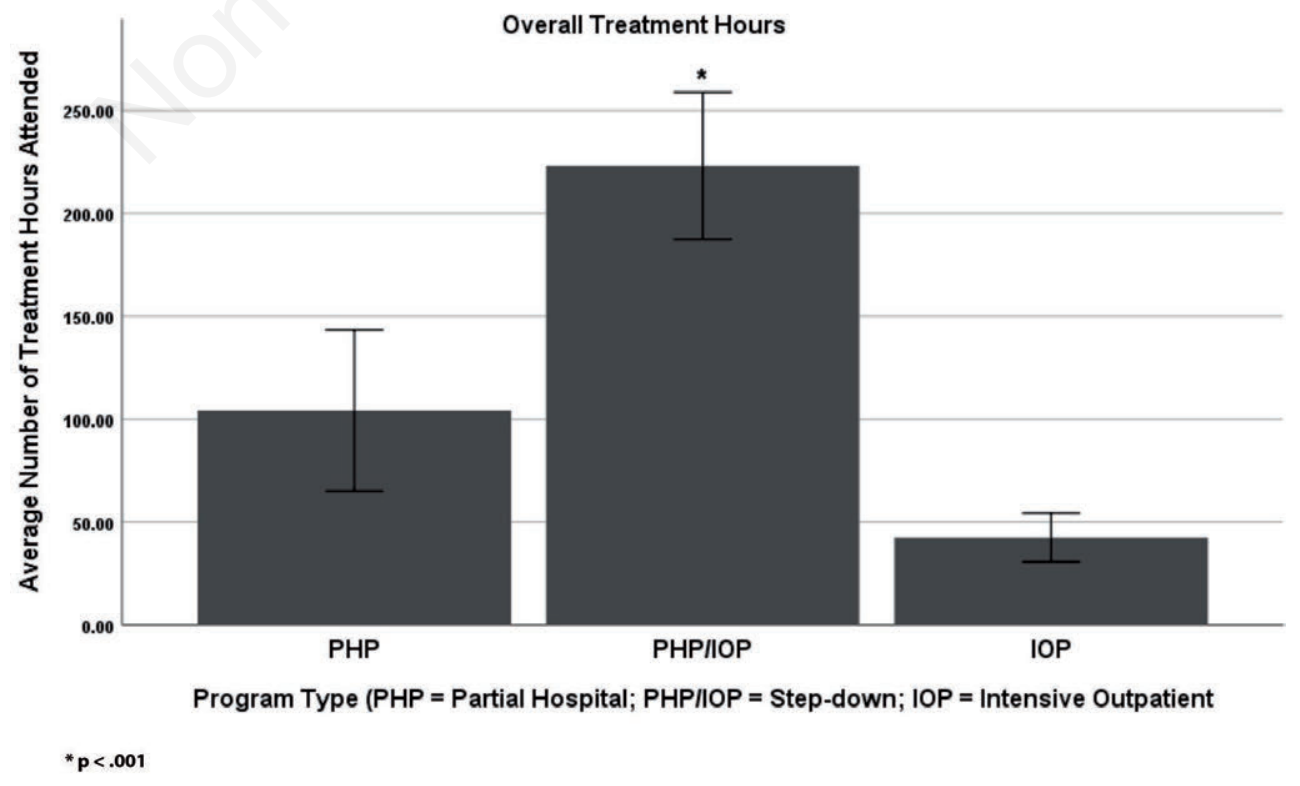

Figure 5. Average Overall Hours in Treatment from Intake to Discharge by Program Type. 
intake were more likely to be placed in the step-down care condition compared to those in the PH program, $F$ (2, $201)=5.61, p<.05$, (Mean difference $=6.94, S E=2.94$, $p=.058$ ), although this analysis only approached significance. There were no other significant results in symptom differences in relation to program type.

\section{Discussion}

Prior study findings support using adapted versions of DBT within PH programs (Lenz \& Del Conte, 2018; Lothes et al., 2016). However, few studies have assessed outcomes in DBT-informed IOP programs, with only preliminary evidence to suggest their effectiveness at reducing various symptoms (Ritschel et al., 2012). The present study findings successfully replicated previous findings from Lothes et al. (2016), showing symptom reduction from intake to discharge with unique clinical measures of depression (CUDOS) and anxiety (CUXOS) in a DBT-informed $\mathrm{PH}$ program as well extended these findings to include assessment of a DBT-informed IOP program and step-down care within these programs.

Specific results of the IOP program mimicked those of the $\mathrm{PH}$ program showing significantly reduced symptom scores from intake to discharge and were consistent with the present study hypotheses. These results were similar to findings of a prior study on a DBT IOP program that showed reductions in depression and anxiety symptoms from intake to discharge (Ritschel et al., 2012). The present study extended prior research findings by showing significant reductions in hopelessness and overall suffering in addition to depression and anxiety from intake to discharge. Moreover, these findings provide some preliminary evidence for the efficacy of adapting DBT to PH and IOP programs and in step-down care conditions, which has been suggested as being efficacious, but not fully examined in prior research studies (Swenson, 2016). In addition, results showed no significant differences in depression, anxiety, hopelessness, and suffering scores based on the program type. It is possible that since both the $\mathrm{PH}$ and IOP programs utilized an adapted version of a comprehensive DBT model, like that of the one described in a study by Lothes et al. (2014), there were no significant differences between groups.

Findings also revealed that patients who attended more overall hours in treatment were also significantly more likely to be in the step-down care condition compared to both the PH and IOP programs. A likely explanation for this finding is that patients who engaged in step-down care may have experienced a greater severity of symptoms at intake compared to those that did not. Theoretically, individuals with more severe symptoms would necessitate more overall hours in treatment to obtain sufficient reductions in symptoms before being discharged from treatment, which has been found in prior studies (Lothes et al., 2016). This explanation was par- tially supported by the study findings regarding the relationship of program placement to symptoms at intake. Results of the present study indicated that individuals in the step-down program had significantly higher depression scores at intake compared to the IOP program. However, there were no other symptom scores that significantly predicted program placement within the sample. It appears that at least in the current sample, attention to a patient's severity of depression symptoms at intake are important when considering program placement and predicting optimal treatment duration within DBT-informed PH and IOP programs. The current study findings indicate that clinicians working in these treatment settings might intentionally screen for depression symptoms at intake as a potential factor in determining program placement.

In addition, a closer examination of the clinical significance of changes in depression and anxiety scores from intake to discharge in relation to program type revealed some interesting findings. Individuals enrolled in only the $\mathrm{PH}$ program and those that utilized step-down care endorsed symptoms corresponding to the moderate to severe range of depression per the CUDOS at intake. Those who engaged in the $\mathrm{PH}$ program endorsed mild depression symptoms at discharge, while those who engaged in stepdown care displayed minimal depression symptoms at discharge. However, both groups mean scores were clustered on the cutoff between mild and minimum depression suggesting that there was not a clinically significant difference in these outcomes. The authors argue that clinicians should consider implementing step-down care as an option due to many patients necessitating a longer duration of treatment that can be obtained through this method. In addition, individual's enrolled only in the IOP program endorsed mild depression symptoms and completed programming with minimal depression symptoms.

A similar trend was revealed for anxiety symptoms on the CUXOS in the sample. Specifically, patients enrolled in the $\mathrm{PH}$ program as well as those who engaged in stepdown care endorsed moderate anxiety symptoms at intake and mild anxiety at discharge. Individuals that began in IOP endorsed moderate anxiety at intake and minimal anxiety at discharge. These findings support prior research that DBT skills groups have more effect on depression than anxiety, as DBT incorporates a significant amount of behavioral activation strategies (Harned et al, 2008). This may also be a function of PTSD symptoms in the sample that were not directly measured on intake or targeted by the PH and IOP curriculum. Regardless, it still seems important to consider symptom score severity upon intake when making program placement decisions.

In addition, overall hours in treatment was not related to any changes in symptom scores in the present study. Prior research has demonstrated that the amount of time spent in treatment is correlated with better overall outcomes, which is partially why comprehensive DBT was originally designed as a one to two-year program (Line- 
han, 1993). However, this relationship was not found within the current study sample. It is possible that the $\mathrm{PH}$ and IOP programs as well as the step-down care condition are equally effective at reducing symptoms of depression, anxiety, hopelessness, and suffering. However, it is more likely that program placement is extremely important in predicting outcomes, not necessarily time in treatment. For example, individuals with higher symptoms of depression at intake may still benefit the most by being placed in a higher level of care (i.e., PH versus IOP programming) with the intention of moving them towards step-down care to increase the likelihood of clinically significant symptom reductions that can be maintaned over a longer period of time. However, individuals with low to moderate symptoms of depression might be best placed in an IOP program which can help them achieve similar symptom reductions in a shorter time frame than engaging in step-down care. The study findings stress the importance of proper placement of individuals into $\mathrm{PH}$ versus IOP programming and offering an option of step-down care based on treatment progress due to stepping down to an IOP program being much cheaper than continuing at a $\mathrm{PH}$ program level.

Overall, the present study findings demonstrate preliminary evidence for adapting DBT within a PH and IOP program. Currently, there is an abundance of empirical evidence for using DBT to treat BPD (Kliem, Kröger, \& Kosfelder, 2010); however, the present findings provide further evidence that DBT-informed programs can be effectively implemented to treat a variety of mental health disorders within unique treatment settings. Moreover, the study findings indicate that it is useful to at least consider step-down care from DBT-informed PH to IOP program for some patients, especially those who present with severe levels of depression symptoms during intake. However, more research is needed to fully understand the possible clinical, financial, and treatment implications of the study findings.

\section{Study Limitations}

The present study used self-report data, which can sometimes be unreliable. Behavioral observations from clinical staff may provide additional insight into treatment outcomes. Unfortunately, internal consistency statistics were unable to be calculated for the symptom measures used in the study; however, reliability and validity have been well established for these measures in other studies examining psychiatric patients. Further, the present study was unable to control for weekly medication management which may have contributed to a portion of the variance in significant findings. Common outcome variables examined in DBT programs such as impulsivity, problematic/suicidal behavior, and emotional dysregulation were not assessed in the present study. Further, only primary diagnoses were obtained for data analysis in the study sample, potentially skewing some results in terms of clin- ical utility for specific patient populations. Moreover, while the heterogeneity of the study sample might be considered a strength due to this being more representative of patients obtaining treatment in community mental health clinics, it also likely creates a limitation in generalizability of findings.

Importantly, there was no control group utilized in the present study; however, the researchers felt it would be unethical to deprive high-risk patients of DBT-informed $\mathrm{PH}$ or IOP programming by placing them on a wait-list control. Furthermore, the study took place in a community-based clinic where neglecting possible referrals of treatment for research purposes would likely result in detrimental effects for patients and treatment providers. Of note, quantitative assignment research designs can be applied to units at various levels (e.g., individuals, community health clinics, neighborhoods, etc.) and are considered a viable alternative to RCT designs (West \& Thoemmes, 2010). These research designs utilize a clinically meaningful assignment variable (e.g., risk level) and can provide a stronger ethical basis for such studies, which was arguably the case in the present study.

\section{Future Directions}

Future research should attempt to replicate the present study by comparing DBT-informed PH and IOP programs as well as step-down care. Of note, fidelity to the original (comprehensive model) was important in the present study's adaptation of DBT to the PH and IOP programs and should be considered when attempting to replicate these findings. In addition, future research might explore other possible mechanisms for symptom reduction in such programs. Perhaps examining mediators/moderators of symptom change such as suicidal behaviors, hospitalizations, and DBT skill acquisition would shed light on specific mechanisms of change. Future studies might also assess coaching phone usage, medication adherence, and mindfulness practice in relation to symptom reduction. Finally, comparing various clinical outcomes across different types, locations, and treatment modalities utilized in various PH and IOP programs would provide a more in depth understanding of the potential treatment and research implications of the present findings.

\section{References}

Antonsen, B. T., Klungsøyr, O., Kamps, A., Hummelen, B., Johansen, M. S., Pedersen, G., ... Wilberg, T. (2014). Stepdown versus outpatient psychotherapeutic treatment for personality disorders: 6-year follow-up of the Ullevål personality project. BMC Psychiatry, 14, 119. doi: 10.1186/ 1471-244X-14-119.

Beck, A. T., \& Steer, R. A. (1988). BHS, Beck Hopelessness Scale: Manual. San Antonio, TX: Psychological corporation.

Ben-Porath, D. D., \& Koons, C. R. (2005). Telephone coaching in dialectical behavior therapy: A decision-tree model for 
managing inter-session contact with clients. Cognitive and Behavioral Practice, 12(4), 448-460.

Bouvard, M., Charles, S., Guerin, J., Aimard, G., \& Cottraux, J. (1992). Study of Beck's hopelessness scale. Validation and factor analysis. L'Encephale, 18(3), 237-240.

Chapman, A. (2019). Borderline personality disorder and emotion dysregulation. Development and Psychopathology, 31(3), 1143-1156. doi:10.1017/S0954579419000658.

Chapman, A. (2019). Phone Coaching in Dialectical Behavior Therapy. New York: Guilford Press.

Eisner, L., Eddie, D., Harley, R., Jacobo, M., Nierenberg, A. A., \& Deckersbach, T. (2017). Dialectical behavior therapy group skills training for bipolar disorder. Behavior Therapy, 48(4), 557-566. doi:10.1016/j.beth.2016.12.006.

Federici, A., \& Wisniewski, L. (2013). An intensive DBT program for patients with multidiagnostic eating disorder presentations: A case series analysis. International Journal of Eating Disorders, 46(4), 322-331. doi:10.1002/eat.22112.

Harned, M. S., Chapman, A. L., Dexter-Mazza, E. T., Murray, A., Comtois, K. A., \& Linehan, M. M. (2008). Treating cooccurring Axis I disorders in recurrently suicidal women with borderline personality disorder: A 2-year randomized trial of dialectical behavior therapy versus community treatment by experts. Journal of Consulting and Clinical Psychology, 76(6), 1068-1075. doi:10.1037/a0014044.

Harned, M. S., Wilks, C. R., Schmidt, S. C., \& Coyle, T. N. (2018). Improving functional outcomes in women with borderline personality disorder and PTSD by changing PTSD severity and post-traumatic cognitions. Behavior Research and Therapy, 103, 53-61. doi: 10.1016/j.brat.2018.02.002.

Jeon, S. W., Han, C., Ko, Y.-H., Yoon, S., Pae, C.-U., Choi, J., ... Zimmerman, M. (2017). A Korean validation study of the Clinically Useful Anxiety Outcome Scale: Comorbidity and differentiation of anxiety and depressive disorders. PLoS ONE, 12(6).

Kliem, S., Kröger, C., \& Kosfelder, J. (2010). Dialectical behavior therapy for borderline personality disorder: a meta-analysis using mixed-effects modeling. Journal of Consulting and Clinical Psychology, 78(6), 936.

Koerner, K. (2012). Guides to individualized evidence-based treatment. Doing dialectical behavior therapy: A practical guide. New York, NY, US: Guilford Press.

Kröger, C., Harbeck, S., Armbrust, M., \& Kliem, S. (2013). Effectiveness, response, and dropout of dialectical behavior therapy for borderline personality disorder in an inpatient setting. Behaviour Research and Therapy, 51(8), 411-416. doi:10.1016/j.brat.2013.04.008.

Lenz, A. S., \& Del Conte, G. (2018). Efficacy of dialectical behavior therapy for adolescents in a partial hospitalization program. Journal of Counseling \& Development, 96(1), 15-26.

Linehan, M. (1993). Cognitive-behavioral treatment of borderline personality disorder. New York, NY: Guilford Press.

Linehan, M. M., \& Chen, E. Y. (2005). Dialectical behavior therapy for eating disorders. In Encyclopedia of cognitive behavior therapy (pp. 168-171). Springer, Boston, MA.

Linehan, M. M., \& Wilks, C. R. (2015). The course and evolution of dialectical behavior therapy. American Journal of Psychotherapy, 69(2), 97-110.

Lothes, J. E., Mochrie, K. D., \& St. John, J. (2014). The effects of a DBT informed partial hospital program on: Depression, anxiety, hopelessness, and degree of suffering. Journal of Psychology and Psychotherapy, 4, 144. doi: 10.4172/21610487.1000144.

Lothes, J. E., Mochrie, K. D., Quickel, E. J. W., \& St. John, J. (2016). Evaluation of a Dialectical Behavior Therapy-informed partial hospital program: Outcome data and exploratory analyses. Research in Psychotherapy: Psychopathology, Process and Outcome, 19(2), 150-156. doi: 10.4081/ripppo.2016.219.

Maffei, C., Cavicchioli, M., Movalli, M., Cavallaro, R., \& Fossati, A. (2018). Dialectical behavior therapy skills training in alcohol dependence treatment: Findings based on an open trial. Substance Use \& Misuse, 53(14), 2368-2385. doi: 10.1080/10826084.2018.1480035.000000000.

Neuhaus, E. C. (2006). Fixed values and a flexible partial hospital program model.

President and Fellows of Harvard College, 14, 1-14. doi: 10.1080/10673220500519706.

Panos, P. T., Jackson, J. W., Hasan, O., \& Panos, A. (2014). Meta-analysis and systematic review assessing the efficacy of dialectical behavior therapy (DBT). Research on Social Work Practice, 24(2), 213-223.

Ritschel, L. A., Cheavens, J. S., \& Nelson, J. (2012). Dialectical behavior therapy in an intensive outpatient program with a mixed $\square$ diagnostic sample. Journal of Clinical Psychology, 68(3), 221-235. doi:10.1002/jclp.20863.

Swenson, C. R. (2016). DBT® Principles in Action: Acceptance, Change, and Dialectics. Guilford Publications.

Stoffers, JM, Vollm, BA, Rucker, G., Timmer, A, Huband, N, Lieb, K (2012). Psychological therapies for borderline personality disorder. Cochrane Database Systematic Review, August 15;8:CD005652.

Tarrier, N., Taylor, K., \& Gooding, P. (2008). Cognitive-behavioral interventions to reduce suicide behavior a systematic review and meta-analysis. Behavior Modification, 32, 77-108.

Telch, C. F., Agras, W. S., \& Linehan, M. M. (2001). Dialectical behavior therapy for binge eating disorder. Journal of Consulting and Clinical Psychology, 69(6), 1061.

Valentine, S. E., Bankoff, S. M., Poulin, R. M., Reidler, E. B., \& Pantalone, D. W. (2015). The use of dialectical behavior therapy skills training as stand-alone treatment: A systematic review of the treatment outcome literature. Journal of Clinical Psychology, 71(1), 1-20. doi:10.1002/jclp.22114.

West, S. G., \& Thoemmes, F. (2010). Campbell's and Rubin's perspectives on causal inference. Psychological Methods, 15(1), 18-37. http://dx.doi.org/10.1037/a0015917.

Zimmerman, M., Chelminski, I., McGlinchey, J. B., \& Posternak, M. A. (2008). A clinically useful depression outcome scale. Comprehensive Psychiatry, 49(2), 131-140.

Zimmerman, M., Chelminski, I., Young, D., \& Dalrymple, K. (2010). A clinically useful anxiety outcome scale. Journal of Clinical Psychiatry, 71(5), 534-42. doi: 10.4088/JCP. 09m05264blu.

Zimmerman, M., \& McGlinchey, J.B. (2008) Patient acceptability of the use of scales to monitor the outcome of depression in clinical practice. Annals of Clinical Psychiatry, 20, 125-129. 